\title{
The LIM-Homeodomain Gene Family in the Developing Xenopus Brain: Conservation and Divergences with the Mouse Related to the Evolution of the Forebrain
}

\author{
Isabelle Bachy, Philippe Vernier, and Sylvie Rétaux \\ UPR 2197 "Développement, Evolution, Plasticité du Système Nerveux," Institut de Neurobiologie Alfred Fessard, Centre \\ National de la Recherche Scientifique, 91198 Gif-sur-Yvette cedex, France
}

A comparative analysis of LIM-homeodomain (LIM-hd) expression patterns in the developing stage 32 Xenopus brain is presented. $x-L h x 2, x-L h x 7$, and $x-L h x 9$ were isolated and their expression, together with that of $x-L h x 1$ and $x-L h x 5$, was analyzed in terms of prosomeric brain development and LIM-hd combinatorial code and compared with mouse expression data. The results show an almost complete conservation of expression patterns in the diencephalon. The $L h \times 1 / 5$ and Lhx2/9 subgroups label the pretectum/ventral thalamus/zona limitans versus the dorsal thalamus, respectively, in alternating stripes of expression in both species. Conversely, strong divergences in expression patterns are observed between the telencephalon of the two species for $L h \times 1 / 5$ and $L h \times 2 / 9$. $L h x 7$ exhibits particularly conservative patterns and is proposed as a medial ganglionic eminence marker. The conservation of diencephalic segments is proposed to mirror the conservative nature of diencephalic structures across vertebrates. In contrast, the telencephalic divergences are proposed to reflect the emergence of significant novelty in the telencephalon (connectivity changes) at the anamniote/amniote transition. Moreover, the data allow the new delineation of pallial and subpallial domains in the developing frog telencephalon, which are compared with mouse subdivisions. In the pallium, the mouse combinatorial expression of LIM-hd is notably richer than in the frog, again possibly reflecting evolutionary changes in cortical connectivity.

Key words: LIM-homeodomain; Xenopus; mouse; pallium; subpallium; prosomere; homology; connectivity
The comparative study of forebrain development was long impaired by difficulties in defining homologous territories between distant species (Striedter, 1997). However, the study of developmental genes has revealed two fundamental aspects of vertebrate brain development.

First, there is a large conservation in the way a topographical organization of structures and connections is set up under the control of genes expressed according to a grid of longitudinal and transverse compartments. As proposed in the prosomeric model, the expression of developmental factors divides the diencephalon into anteroposterior segments that prefigure adult functional units (Puelles and Rubenstein, 1993; Puelles, 1995). The principles of this model are verified in the vertebrate phylum: prosomeric subdivisions are reported in lamprey [vertebrate agnathe (Pombal and Puelles, 1999)], zebrafish [gnathostome (Wullimann and Puelles, 1999; Hauptmann and Gerster, 2000)], Xenopus [tetrapod (Milan and Puelles, 2000)], chick [amniote (Figdor and Stern, 1993)], and mouse (mammal), five species representing major transitions in vertebrate evolution. Such prosomeric (transverse/anteroposterior) subdivisions are more controversial in the telencephalon. Nevertheless, the expression of major regulators (Dlx-2, Pax -6, Emx -1) along the telencephalic dorsoventral axis also reveals strong similarities of molecular profiles between

\footnotetext{
Received March 13, 2001; revised June 28, 2001; accepted July 16, 2001.

This work was supported by AFIRST, Centre National de la Recherche Scientifique, and Lilly Foundation. Thanks to Agustin Gonzales for interesting discussions. Correspondence should be addressed to Dr. Sylvie Rétaux, UPR 2197 "Développement, Evolution, Plasticité du Système Nerveux," Institut de Neurobiologie Alfred Fessard, Centre National de la Recherche Scientifique, Avenue de la Terrasse, 91198 Gif-sur-Yvette cedex, France. E-mail: Sylvie.Retaux@iaf.cnrs-gif.fr. Copyright (C) 2001 Society for Neuroscience 0270-6474/01/217620-10\$15.00/0
}

mouse and chicken pallium/subpallium (Puelles et al., 2000). Therefore, subdivisions and field homologies in the telencephalon are also postulated.

Second, there are significant differences in the relative size of cerebral areas, in the connections between areas, and in neuronal phenotypes among vertebrates, especially in the forebrain. A major evolutionary trend is the progressive involvement of the cortex in the processing of thalamic sensory information in tetrapods (Marin et al., 1998a; Reiner et al., 1998). Unknown changes in cell specification gene expression patterns must have allowed this functional diversification, although the general Bauplan to build a brain has been conserved across vertebrates. As emphasized by Striedter (1997), a major step in increasing the number and complexity of forebrain connections was achieved at the anamniote/amniote transition.

To approach the question of the evolutionary changes in regionalization and connectivity in vertebrate forebrain, we used LIM-homeodomain (LIM-hd) factors as functional determinants of cell identity. They govern not only regional specification, but also axonal projection patterns and neurotransmitter phenotypes, by using a LIM-hd combinatorial code well described in the spinal cord (Jessel, 2000). Moreover, LIM-hd gene function in neural development seems to be conserved across phylogeny (Hobert and Ruvkun, 1998). Therefore, discrete changes in regional and/or combinatorial LIM-hd expression would be susceptible to alter connectivity patterns between areas and to be selected in the evolutionary process. Here, we used the mouse/Xenopus comparison to analyze differences in LIM-hd expression domains between anamniotes and amniotes, and we tried to correlate these changes with known changes in morphogenesis and connectivity of forebrain structures. The $L h \times 1 / 5$, $L h \times 2 / 9$, and $L h \times 6 / 7 / 8$ sub- 
groups were selected for their predominant forebrain expression in mouse (Rétaux et al., 1999). We isolated new Xenopus orthologs $(x-\operatorname{Lh} x 2, x-\operatorname{Lh} x 7$, and $x-\operatorname{Lh} x 9)$, we analyzed their expression together with that of $x-L h x 1 / 5$ in terms of both prosomeric organization and combinatorial LIM-hd expression, and we compared the resulting patterns with the mouse.

\section{MATERIALS AND METHODS}

RT-PCR cloning. Total RNA from brains of stages 32 or 39/40 embryos were reverse transcribed to cDNA with avian myeloblastosis virus reverse transcriptase (Boehringer Mannheim, Mannheim, Germany) and used as templates for PCR reactions (Qiagen, Hilden, Germany) using the following oligonucleotide primers: for $L h x 7 / 8$, Fdeg78: AARGTIAAYGAYYTITGYTGGCAYGT and Rdeg78: TGICKIGCICKRCARTTYTGRAACCA; for $L h \times 2 / 9$, Fdeg9: TIGCIGTIGAYAARCARTGGCAY(ACT)T and 32, MAYTTIGCYCTIGCRTTYTGRAACCA (where I is an inosine residue); for $L h x 1$ and $L h x 5$, FX1: TGCCTTCTATTCTCCTAATCCGCCC; RX1: CAGCTTAGGCTACCACACTGCCG; FX5: GGATTTCACTGGACTTGGCTTCTGC and RX5: GTTGGAATCAGGCGTACAAGCACC. The various primer combinations led to the amplification of single bands. After these fragments were subcloned (700 bp to $1 \mathrm{~kb}$ ) into pGEM-T (Promega, Madison, WI), sequencing of several independent clones revealed the presence of various fragments. Analysis and alignments performed using the ClustalX program identified the new clones as the Xenopus orthologs of mouse $\operatorname{Lh} x 2, \operatorname{L} h x$, and $\operatorname{Lh} x 7$ genes (GenBank accession numbers AJ311711, AJ311712, AJ311713, AJ311714, and AJ311715). Xenopus and mouse Lhx1 and Lhx5 were reisolated using primers designed in the already published sequences (Taira et al., 1992; Fujii et al., 1994; Toyama et al., 1995; Sheng et al., 1997). The $x$-Dll3 plasmid was a gift of Nancy Papalopulu (Cambridge, UK).

In situ hybridization. The pGEM-T plasmids were linearized with restriction enzymes NdeI or NcoI (Promega) and used as template for RNA synthesis with T7 or SP6 polymerase (Stratagene, La Jolla, CA) in the presence of digoxigenin-11-UTP (Boehringer Mannheim) for antisense and sense control probes, respectively. Xenopus or mouse embryos were fixed overnight in MEMFA (0.1 M MOPS, pH 7.4, 2 mM EGTA, 1 $\mathrm{mM} \mathrm{MgSO}_{4}, 3.7 \%$ formaldehyde) at $4{ }^{\circ} \mathrm{C}$ and then progressively dehydrated in methanol and stored at $-20^{\circ} \mathrm{C}$ until use. After rehydration, embryos were immersed in MEMFA at room temperature for $10 \mathrm{~min}$ and bleached in $6 \% \mathrm{H}_{2} \mathrm{O}_{2}$ for $1 \mathrm{hr}$. Embryos were treated with proteinase $\mathrm{K}(10 \mu \mathrm{g} / \mathrm{ml}, 15 \mathrm{~min})$ and fixed at room temperature for $20 \mathrm{~min}$ before prehybridization $\left(1 \mathrm{hr}\right.$ at $65^{\circ} \mathrm{C}$ and $2-4 \mathrm{hr}$ at $\left.55^{\circ} \mathrm{C}\right)$. Hybridization was performed overnight at $55^{\circ} \mathrm{C}$ in a $50 \%$ formamide hybridization medium containing $1 \mu \mathrm{g}$ of RNA probe. Hybridization was detected using an alkaline phosphatase-coupled anti-digoxigenin antibody (Boehringer Mannheim) diluted to 1:1500. Alkaline phosphatase staining was developed with NBT/BCIP (Boehringer Mannheim).

Two-color whole-mount in situ hybridization was performed with differently labeled RNA probes (fluorescein-UTP and digoxigeninUTP), and subsequent visualization of transcripts in red and purple was obtained with INT/BCIP or NBT/BCIP (Boehringer). For histological observation, labeled embryos were embedded in gelatin/albumin and vibratome sectioned at $25 \mu \mathrm{m}$. Photographs were taken on a Leica microscope, scanned, and mounted for figures with Adobe Photoshop (images were corrected for brightness/contrast or cropping, but no other correction was made)

Immunohistochemistry. To compare gene expression patterns with the position of primary axon tracts, in situ hybridization was combined with immunohistochemistry using a monoclonal antibody against $\alpha$-acetylated tubulin (Sigma, St. Louis, MO). In situ hybridization was performed first as described above, brains were dissected out, and classical immunofluorescence staining was performed (primary antibody dilution 1:700).

\section{RESULTS}

The Xenopus orthologs of mouse Lhx1/2/5/7/9, named $x$-Lhx1/2/ $5 / 7 / 9$, respectively, were isolated by degenerated or classical PCR, and their orthology relationships were assessed after alignments with ClustalX and distance method analysis (data not shown). All the sequences that we isolated fit well with the molecular phylogeny of the vertebrate LIM-hd factors (Failli et al., 2000). No divergent sequences have been found that might indicate the existence of additional members of the LIM-hd family, as a possible result of the partial genome tetraploidization that is known to have occurred in Xenopus laevis. We have also searched for additional genes with a PCR-based approach on cDNA and genomic DNA and have not been able to demonstrate the existence of specific paralogs in Xenopus, neither in the $x$-Lhx2/9 nor in the $x$-Lhxl/5 families.

For the sake of comparison with observations made in mouse mostly at embryonic day (E) 12.5 and E13.5, where neurogenesis is at its peak, regional specification is well advanced, and connectivity begins to be established in pioneering axonal pathways, we used Xenopus embryos at stages 32 and 39/40, in which similar events can be detected. The results were basically identical and therefore are presented on stage 32 embryos.

\section{$\boldsymbol{x}$-Lhx7 expression is well conserved}

In mouse, the $L h x 6 / 7 / 8$ subgroup shows the most restricted brain expression among the LIM-hd family, being expressed in the medial ganglionic eminence (future pallidum) and the hypothalamus (Grigoriou et al., 1998) (Fig. 1D). For comparison purposes we used the Xenopus ortholog of $\operatorname{Lh} x 7$. In the brain of stages 32 and 39/40 embryos, $x-L h x 7$ showed two main expression domains (Fig. 1A): a large, triangle-shaped domain at the ventral/anterior base of the telencephalon, just anterior to the optic recess, and a long thin band of expression in the diencephalon that followed the longitudinal axis of the brain. A small cluster of $x-\operatorname{Lh} x 7$ expressing cells was also consistently found in the ventroposterior part of the diencephalon (Fig. $1 A, B$, arrows). To establish whether these expression domains were homologous to the situation in mouse, and because distalless genes are established markers of the medial and lateral ganglionic eminences and of the telencephalic stalk region (Fig. $1 D$ ) in all studied vertebrate species, we performed double labeling with $x$-Dll3. The telencephalic domain of $x-L h x 7$ expression was included into the $x$-Dll3 expression area and ran along the anterior border of the optic stalk (Fig. $1 B, C$ ), suggesting that it could represent Xenopus medial eminence and aep/poa. Mouse $\operatorname{Lh} x 7$ is not expressed in the aep/poa (Fig. $1 D$ ), but its paralog $m$-Lhx6 is (Lavdas et al., 1999). Thus, $x$-Lhx7 expression in the ventral telencephalon is identical to that of its mouse paralogs. The diencephalic expression territories of $x-L h x 7$ and $x-D l l 3$ were also closely related (Fig. 1D). As deduced from careful observations in toto and on sections, the most anterior part of their expression was colocalized, whereas in the dorsal aspect the thin band of $x-L h x 7$ expression ran parallel to the larger band of $x-D l l 3$ expression (the ventral border of $x$-Dll3 expression defines the alar/basal boundary). This $\mathrm{x}$-Lhx7-positive, $x$-Dll3-negative domain therefore might correspond to the mammilary area. Moreover, $\mathrm{x}-L h x 7$ was expressed in branchial arches and jaws (Fig. $1 C$ ), just like its mouse ortholog. These observations showed that $\operatorname{Lh} x 7$ overall expression was well conserved between mouse and frogs and that $x-\operatorname{Lh} x 7$ might be considered as a marker of Xenopus medial ganglionic eminence, telencephalic stalk, and hypothalamus.

\section{$x-L h \times 2$ and $x-L h x 9$ in pallial and subpallial territories of the telencephalon}

Next we sought to integrate the two paralogs of the more complex $L h \times 2 / L h x 9$ subgroup into this scheme. In mouse, $L h \times 2$ labels the entire telencephalon (pallial and subpallial territories) and the hypothalamus, whereas $\operatorname{Lh} x 9$ expression is strictly pallial and is included in the Lhx2-expressing domain (Rétaux et al., 1999) (Fig. 2H). Moreover, $L h x 2$ and $\operatorname{Lh} x 9$ are expressed in p4 and p2 
Figure 1. Lhx7 seems to be a general medial ganglionic eminence marker. $A$, $B$, Lateral whole-mount views of stage 32 brains labeled for $x-L h x 7$ expression $(A)$ and double labeled for $x$-Lhx7 and $x$-Dll3 $(B)$. Colors for double labeling are indicated on each panel. The arrow points to the diencephalic group of cells discussed in Results. $C$, Transverse anteroposterior sections of an embryo double labeled for $x-L h x 7$ and $x$-Dll3. The orientation of sections is indicated by the white line in B. Arrowheads in $B$ and $C$ indicate the boundaries between ventral and dorsal telencephalon (vtel and $d t e l)$ and between ventral and dorsal thalamus ( $v t$ and $d t$ ), as revealed by $x$-Dll3 staining. $e$, Eye; $b a$, branchial arches; $c g$, cement gland; hyp, hypothalamus. D, Schematic color-coded (see box) comparison of Xenopus and mouse Lhx7 expression patterns, with reference to $x$-Dll3 (to be compared with Dlx1/2/5/6 of the mouse). Note the strong conservation of patterns. For mouse, $L h x 7$ expression is drawn after Grigoriou et al. (1998). aep/poa, Anterior entopeduncular/preoptic area; $c b$, cerebellum; $d t$, dorsal thalamus; $d t e l$, dorsal telencephalon; emt/spv, eminentia thalami/supraoptic paraventricular area; is, isthmus; lge, lateral ganglionic eminence; $m a$, mammilary area; $m g e$, medial ganglionic eminence; mes, mesencephalon; met, metencephalon; or/os, optic stalk/recess; $p t$, pretectum; $p 1-p 4$, prosomeres 1-4; tec, tectum; $t u$, tuberal area; $v t$, ventral thalamus; vtel, ventral telencephalon.
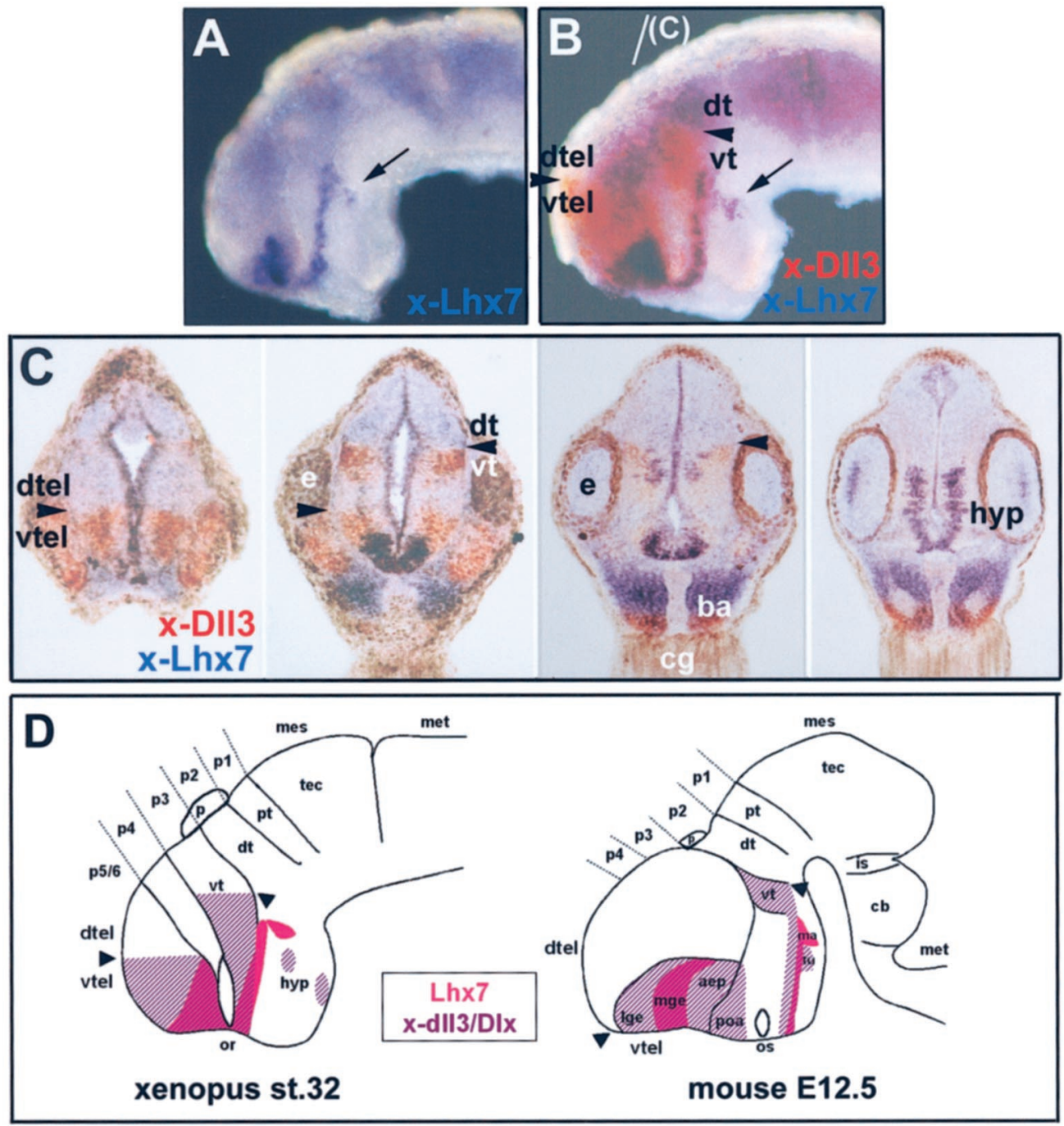

prosomeres in mouse and show extensive expression throughout the pretectum and tectum.

The two Xenopus paralogs $x$-Lhx2 and $x$-Lhx9 showed spectacular, striped-like patterns in the developing Xenopus brain (Fig. $2 A, B)$. In the telencephalon, $x-L h x 9$ expression domain was more restricted than $x$-Lhx2, and double labeling was used to position their respective domains (Fig. $2 C$ ). The rather ventral $x$-Lh $x 9$ positive domain (Fig. 2C, arrow) was included in the large $x$-Lhx2-positive area that covered almost the entire extent of the telencephalon. The situation was similar therefore to that found in rodents in terms of the extent of expression (broad $x$-Lhx2 domain, more restricted $x$-Lhx 9 domain). However, it looked different from the mouse in terms of pallial/subpallial expression: in Xenopus, $x$ - $\operatorname{Lh} x 9$ was found anteroventrally, whereas it is strictly pallial in mouse. Another difference with the rodent is that $x$-Lhx2 did not cover the most basal part of the telencephalon (Fig. 2H, mge, aep, poa): $x$-Lhx7 and $x$-Lhx2 expression were mutually exclusive (Fig. $2 F$, summary on Fig. $2 H$ ).

To further investigate this question, and because the functional telencephalic divisions of the developing Xenopus are poorly known and delimited, we next asked whether $x$ - $\operatorname{Lh} x 9$ (contrarily to its mouse ortholog) was indeed expressed in the subpallium by double labeling for $x$ - $\operatorname{Lh} x 7$ (expressed in the medial ganglionic eminence) or $x$-Dll3 (a general ganglionic eminence, subpallial marker; see above). As observed in toto on Figure $2 D$ and confirmed on sections in Figure $2 E$, the $x-L h x 9$ telencephalic domain (Fig. 2E, arrow) was included in the $x$-dll3 domain, suggesting that it labeled a part of the future basal ganglia. Moreover, the $x$ - $\operatorname{Lh} x 9$ domain was strictly adjacent and did not overlap with the $x$-Lhx7 domain (Fig. $2 G$ ), suggesting that it did not label the medial eminence (future pallidum) but rather another subdivision of the subpallium. Finally, the dorsalmost telencephalic region that expressed $x$-Lhx2, but neither $x$-Lh $x 9$ nor $x$-Dll3, had to be considered as pallial. Therefore, the $x$-Lhx2/xLhx 9 situation was suggestive of a partial inversion of expression patterns between the two paralogs as compared with mouse. This was also supported by the fact that $x-\operatorname{Lh} x 2$ (but not $x$-Lh $x 9$ ) was expressed in the frog pineal gland (Fig. $2 A, p$ ), whereas the case is strictly the opposite in mouse (Rétaux et al., 1999). Nevertheless, the region of the frog basal telencephalon defined by $x-\operatorname{Lh} x 7$ expression (mge, aep/poa) did not express a member of the Lhx2/9 subgroup, which constitutes a major difference with the mouse. These results are summarized on Figure $2 H$.

\section{$x-L h \times 2$ and $x-L h x 9$ in diencephalon and mesencephalon}

$x-\operatorname{Lh} x 9$ and $\mathrm{x}-\operatorname{Lh} x 2$ were expressed as bands with extremely sharp borders in the diencephalon and mesencephalon. Double labeling 

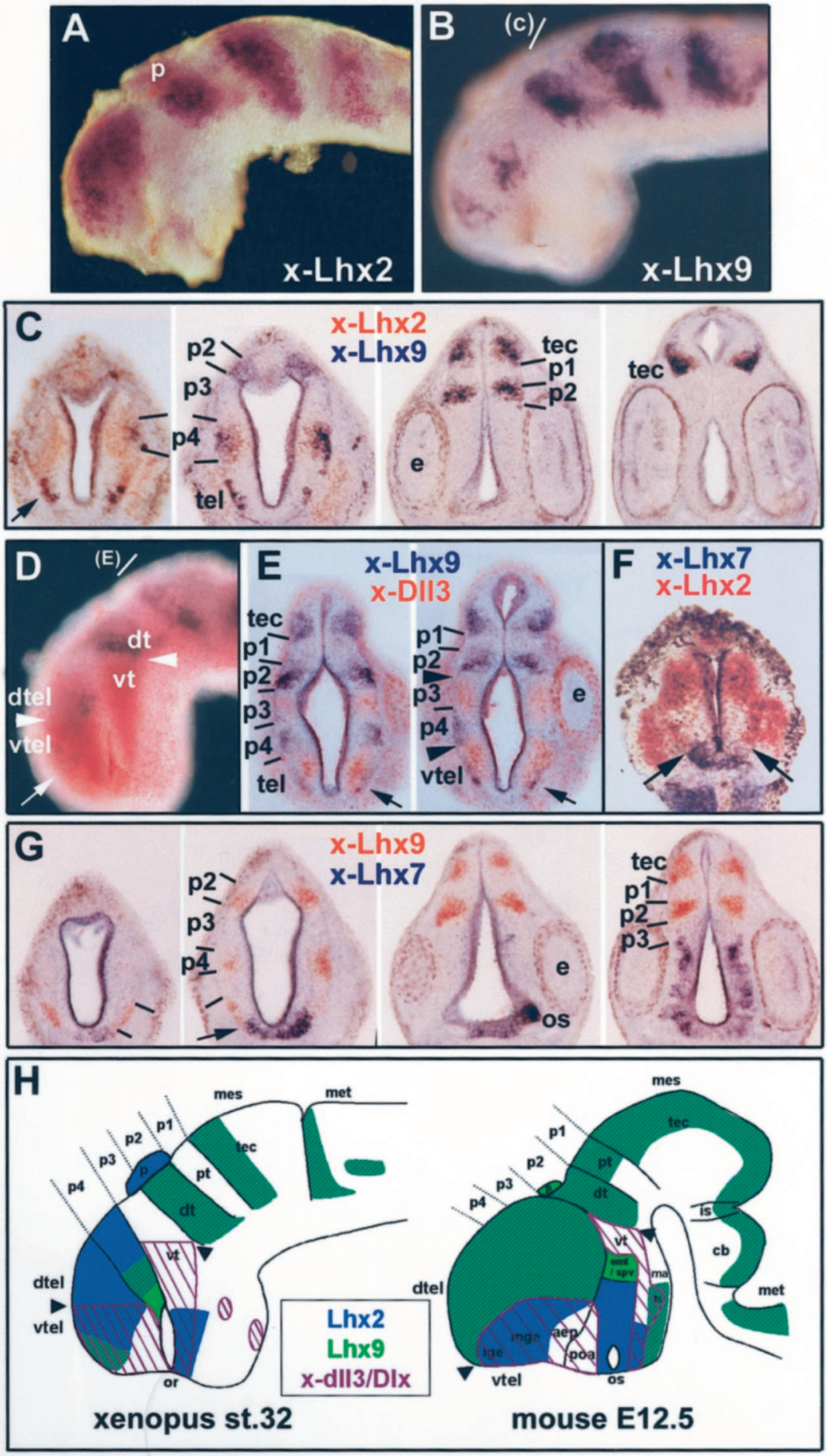

Figure 2. Telencephalic expression of $L h x 2$ and $L h x 9$ shows major differences between Xenopus and mouse. $A$, $B$, Lateral whole-mount views of stage 32 brains labeled for $x$ - $\operatorname{Lh} \times 2(A)$ and $x-\operatorname{Lh} x 9(B)$ expression. $C$, Transverse anteroposterior sections of an embryo double labeled for $x$-Lhx2 (orange) and $x$-Lhx9 (purple). The orientation of sections is indicated by the white line in $B$. The arrow points to the ventral telencephalic domain of $x$ - $L h x 9$ expression. $D, E$, Whole-mount $(D)$ and anteroposterior transverse sections $(E$, orientation given by white line in $D$ ) of double labeled $x$-Lhx 9 (purple) and $x$-Dll3 (orange) embryos. Arrows point to the ventral telencephalic domain of $x-L h x 9$ expression, and arrowheads indicate the boundaries between ventral and dorsal telencephalon (vtel and dtel) and between ventral and dorsal thalamus $(v t$ and $d t$ ). F, A section through the telencephalon of an embryo double labeled for $x-L h \times 2$ (orange) and $x-L h x 7$ ( purple). Arrows point to the nonoverlapping expression of the two genes in the basal forebrain. $G$, Anteroposterior transverse sections of double-labeled $x-\operatorname{Lh} x 9$ (orange) and $x-L h x 7$ (purple) embryos. The arrow points to the sharp boundary between the two expression domains in the ventral telencephalon. $H$, Schematic color-coded (see box) comparison of Xenopus and mouse Lhx2/9 expression patterns, with reference to $x$-Dll3/mouse Dlx. Mouse Lhx2/9 expression is drawn after Rétaux et al. (1999). Note the major differences, especially in the telencephalon of the two species. In particular, $x-\operatorname{Lh} x 9$ is subpallial whereas $m-L h x 9$ is pallial, and $x$-Lhx2 does not cover the entire telencephalon whereas $m-L h x 2$ does. See Figure $1 D$ for abbreviations. with $x$-Dll3, expressed in the diencephalic p3 prosomere (including the ventral thalamus) showed that $x-L h \times 2 / 9$ were expressed just anterior to $\mathrm{p} 3$, in $\mathrm{p} 4$, and just posterior to p3, in p2 (dorsal thalamus)(Fig. 2D,E). The two paralogs therefore labeled $\mathrm{p} 4$ and p2 in frog and showed conservation of expression in most of the diencephalon as compared with mouse, including a ventral diencephalic/hypothalamic band of staining for $x$-Lhx2 (Fig. 2A). By contrast, we again observed differences in regions posterior to $\mathrm{p} 2$. First, $x-\operatorname{Lh} x 2$ and $x-\operatorname{Lh} x 9$ were not expressed in the pretectum (prosomere $\mathrm{p} 1$ ). This point was established by double labeling for $\alpha$-acetylated tubulin to position the tract of the posterior com- missure (TPC), one of the major early tracts that grows through prosomere $\mathrm{p} 1$, with regard to $x-L h \times 2 / 9$ stripes of expression. The TPC ran just between the two $x$ - $L h \times 2 / 9$-expressing stripes of $\mathrm{p} 2$ and mesencephalon, respectively (see Fig. $5 A$ ). Second, in the mesencephalon itself, $x-L h x 2$ and $x-L h x 9$ were absent in the posterior tectum of the frog, as can also be defined by tubulin staining and morphological observation of the isthmus.

Finally, sections through the brains of embryos double labeled for $x$ - $\operatorname{Lh} x 2$ and $x-\operatorname{Lh} \times 9$ showed a perfect colocalization of the two paralogs in expressing bands of the diencephalon and mesencephalon (Fig. $2 C$ ), except in the dorsal part of $\mathrm{p} 4$. The results of the 

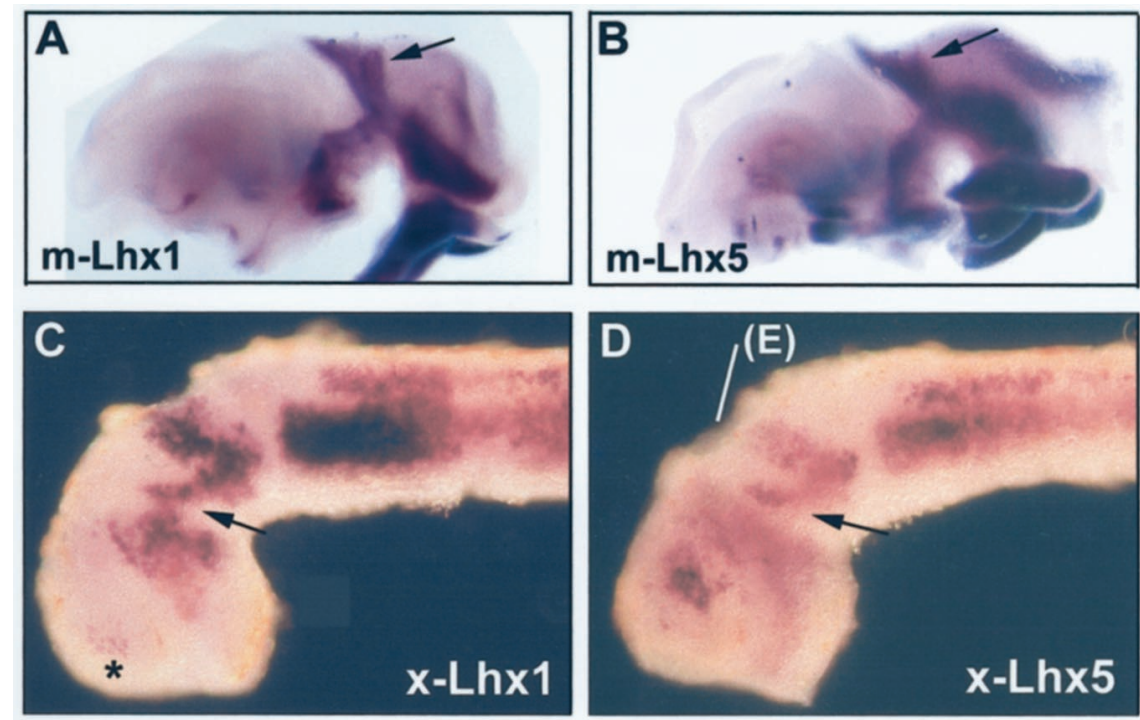

Figure 3. Lhxl and Lhx5 label the zona limitans intrathalamica, and their diencephalic expression patterns are conserved between Xenopus and mouse. $A-D$, Whole-mount lateral views of E12.5 mouse embryos $(A, B)$ and stage 32 Xenopus embryos $(C, D)$ stained for $m / x$-Lhx1 and $m / x L h x 5$, as indicated on panels. In $A$ and $B$, arrows indicate the strong labeling in the pretectum $(p 1)$. In $C$, the asterisk points to the ventral telencephalic domain of $x$-Lhxl expression, and the arrows in $C$ and $D$ indicate the thin band of expression that surrounds the dorsal thalamus and is suggested to label the zona limitans intrathalamica (zli). E, Anteroposterior transverse sections (orientation given by white line in $D$ ) of double-labeled $x$-Lhx1 (orange) and $x$-Lhx5 (purple) embryos. The arrow points to the thin band of expression of the two genes that envelops ventrally the dorsal thalamus $(p 2)$ and is suggested to be the zli. $F$, Two transverse sections through the telencephalon and diencephalon of $x$-Lhx5 (purple) and $x$-Dll3 (orange) double-labeled embryos. Arrowheads indicate the boundaries between ventral and dorsal telencephalon ( $\mathrm{vtel}$ and $\mathrm{dtel}$ ) and between ventral and dorsal thalamus ( $v t$ and $d t$ ). Arrow points to zli. Note that the thin band/zli is juxtaposed but not double labeled with $x$-Dll3. The dotted line marks the limit between dorsal p3 (expressing $x-L h x 1 / 5$ ) and ventral $\mathrm{p} 3 /$ hypothalamus (expressing only $x$-Dll3). $G$, Two coronal hemisections (in the same plane of section) through the telencephalon of an embryo labeled for $x$-Lhx1 (left) or $x$-Lhx 9 (right) to show that the ventral telencephalic $x-L h x 1$ domain is included in the $x$ - $L h x 9$ domain. $H$, Schematic colorcoded (see box) comparison of Xenopus and mouse Lhx1/5 expression patterns, with reference to $x$-Dll3/ mouse Dlx. The diencephalic conservation can be opposed to the telencephalic divergences between the two species. For abbreviations, see legend to Figure
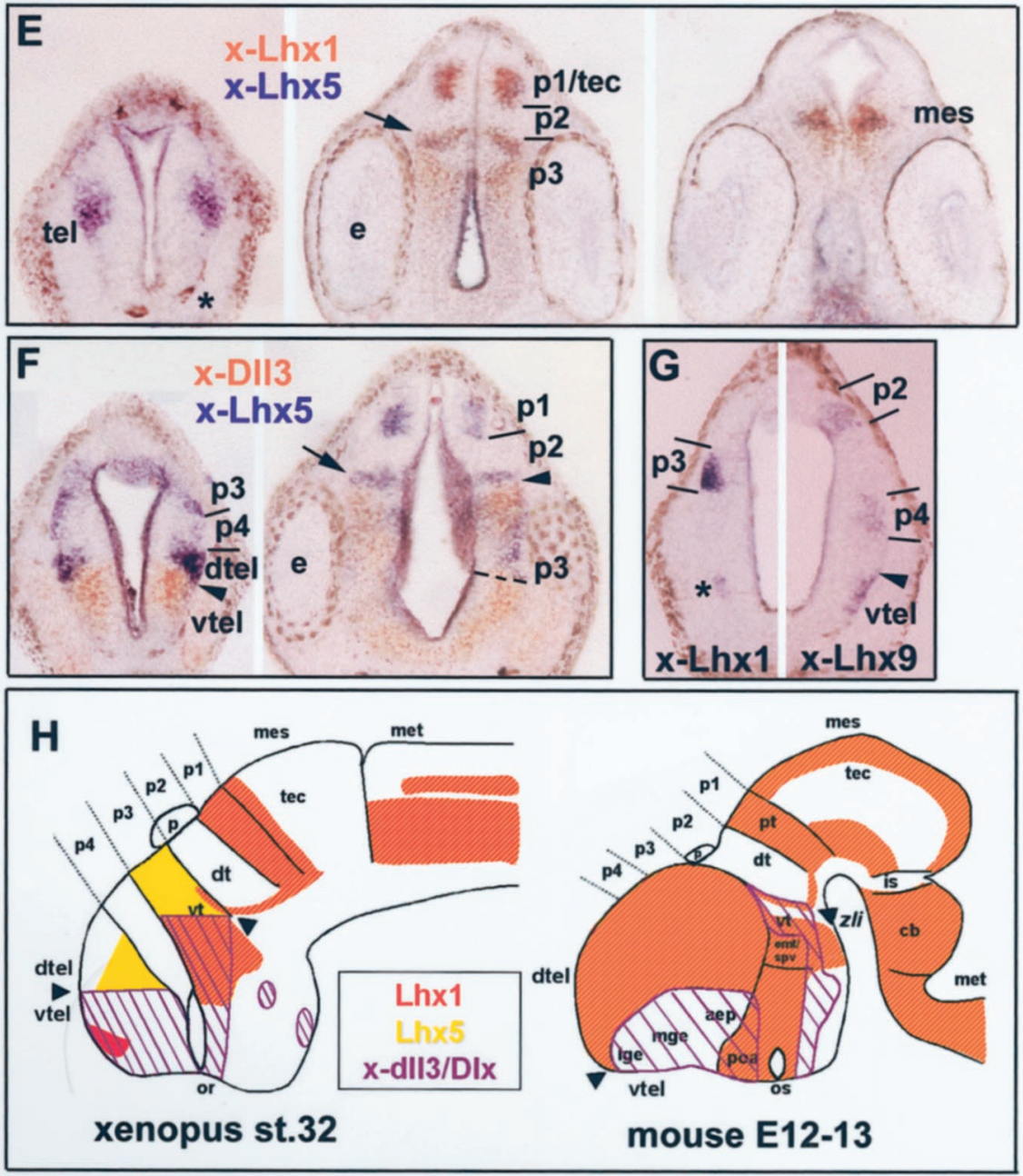

Xenopus/mouse comparison for $L h \times 2 / 9$ are recapitulated in Figure $2 H$.

\section{Lhx1 and $L h \times 5$ subgroup}

Because mouse expression data for these two paralogs were not available in a precise manner, we first reexamined their expression patterns in E12.5 and E13.5 mouse brains (Fig. 3A,B). Detailed data will be available elsewhere (S. Rétaux and I. Bachy, unpublished observations), and results compiling the expressions at E12.5 and E13.5 are summarized on Figure $3 H$ (right panel). Both genes are expressed in the pallium, the hypothalamus, the ventral thalamus (p3), the zona limitans intrathalamica (zli), the pretectum, and the tectum. Interestingly, $m L h x 1 / 5$ are absent in the developing basal ganglia and dorsal thalamus (p2) of the rodent. 

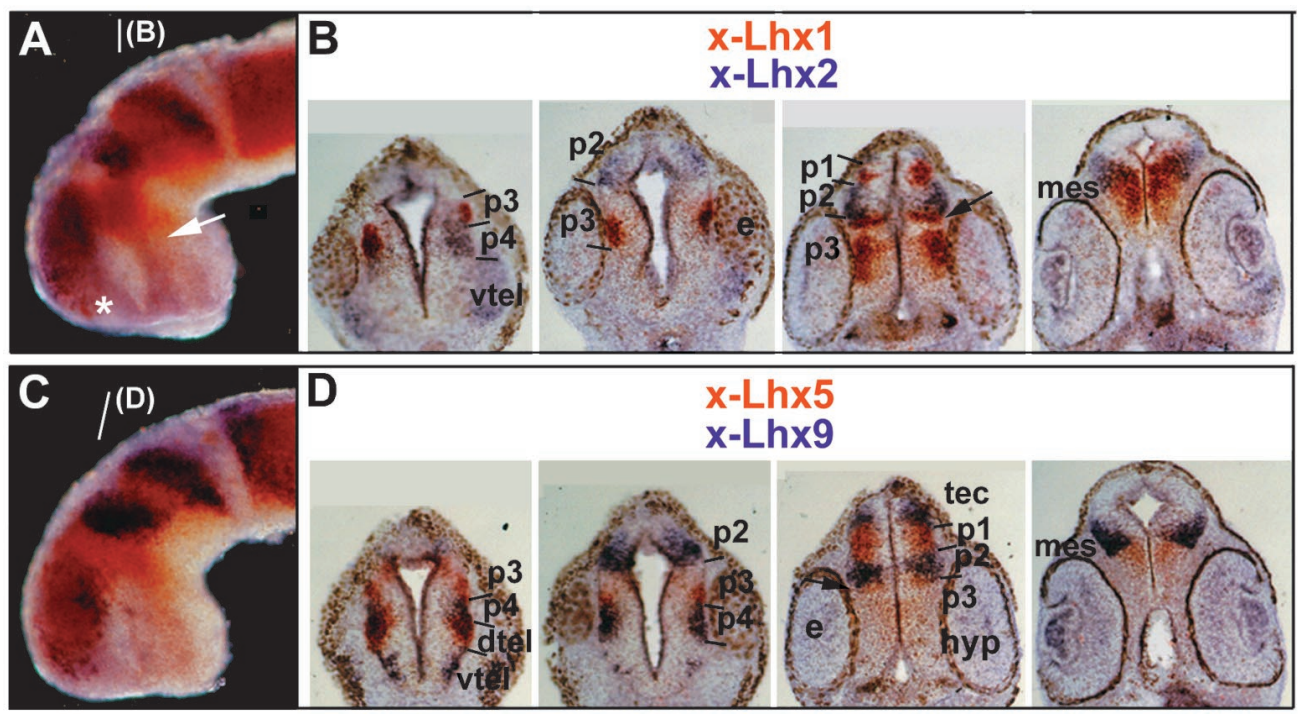

Figure 4. $x$-Lhx1/5 and $x-L h x 2 / 9$ expression domains are almost exclusive. $A, B$, Whole-mount brain $(A)$ and transverse sections $(B$, orientation given by white line in $A$ ) of embryos double labeled for $x$-Lhx1 (orange) and $x$-Lhx2 (purple). The arrow in $A$ points to the boundary between ventral diencephalic $x-L h x 2$ expression and the more dorsal $x$-Lhx1 domain. Note that except for the ventral telencephalic domain where both genes are expressed (asterisk in $A$ ) and the anterior tectum, the expression domains are exclusive. $C, D$, Whole-mount brain $(C)$ and transverse sections (D, orientation given by white line in $C$ ) of embryos double labeled for $x$-Lhx5 (orange) and $x$-Lhx9 (purple). Note that except for the anterior tectum, the expression domains are exclusive. The arrows in $B$ and $D$ indicate the thin band of $x$-Lhx1/5 expression that envelops ventrally the $L h x 2 / 9$-expressing dorsal thalamus $(p 2)$. We suggest that this could represent the zona limitans intrathalamica.

\section{$x-L h \times 1$ and $x-L h \times 5$ in the telencephalon}

Xenopus orthologs showed rather complex expression patterns (Fig. 3C,D). Both $x$-Lhx1 and $x$-Lhx5 showed a single (but distinct) expression domain in the telencephalon. The $x$-Lhx1positive area (Fig. 3C,E, G, asterisks) was modest, located in the subpallium, and included in the $x-L h x 9$-positive region (Fig. $3 G$ ). $x-L h x 5$, on the other hand, labeled a large band in the telencephalon that was juxtaposed dorsally to the $x$-Dll3-defined subpallium (Fig. $3 F$ ). The ventral border of $x$ - $L h x 5$ domain would thus follow the palliosubpallial border. The telencephalic $x$-Lhx5 domain did not cover the entire pallium (particularly, the dorsal aspect of the pallium was not labeled) and was included in the $x$-Lhx2-positive area (data not shown). $x$-Lhx5 labeling did not cross the telencephalic/p4 border, as shown by examination of $x$-Lhx $9 / x-L h x 5$ double-labeled brains and sections (Fig. 4C,D). Therefore, we concluded that $x-L h x 5$ specifically labeled a subdivision of the pallium in Xenopus embryos (Fig. $3 H$, summary).

\section{$x-L h \times 1$ and $x-L h \times 5$ in the diencephalon and mesencephalon}

In more posterior regions, $x$-Lhx1 and $x-L h x 5$ colocalized in the majority of their expression domains. Both genes were expressed in prosomere p3 (ventral thalamus) (Fig. $3 E$ ), as shown with $x$-Dll3 double staining (Fig. $3 F$ ). $x$-Lhxl was expressed much higher and stopped at the dorsal boundary of the $x$-Dll3 domain, whereas $x-L h x 5$ ran more dorsally, resulting in the entire covering of dorsal p3. Both genes appeared to cross the alar/basal boundary (defined by the ventral border of the $x$-Dll3 diencephalic band) and to be expressed in a region of the hypothalamus (Fig. $4 B-D)$. Finally, in the ventral diencephalon the $x-L h x 1 / x-L h x 5$ domain abutted the $x$-Lhx2 domain (Fig. 4B, arrow).

As shown by double labeling between the paralogs of the $x$-Lhx1/5 and $x$-Lhx2/9 families, neither $x$-Lhx1 nor $x$-Lhx5 was expressed in the dorsal thalamus itself, but rather enveloped this structure with a strong expression in the pretectum (prosomere p1) and with a thin band of expression on the ventral and anterior side (Figs. 3, 4, arrows). Because this thin band was just dorsal to $x$-Dll3 but did not express $x$-Dll3 (Fig. 3F, arrowhead), and because it was just adjacent to $x-L h x 2 / 9$ expressing dorsal thalamus (Fig. $4 B-D, p 4$, arrows), we suggest that this could represent the zona limitans intrathalamica. Moreover, the pretectal expression of $x-L h x 1 / 5$ was established by double labeling for the TPC with $\alpha$-acetylated tubulin (Fig. 5B). Finally, the two paralogs were present in a thin band of the anterior mesencephalon, where they overlapped with $x$-Lhx2/9 expression (Fig. $4 B-D$ ). In summary (Fig. $3 H$ ), the mouse/frog comparison seems to indicate a good degree of conservation of expression in the pretectum, thalamus, and hypothalamus, but differences are found in the telencephalon, where the combinatorial expression of the two paralogs is notably different between the two species.

\section{DISCUSSION}

\section{Xenopus/mouse LIM-hd expression: general conservation but telencephalic divergences}

Our data suggest two general remarks. First, the current view for a common Bauplan of brain development among vertebrates fits well with our Xenopus/mouse comparison of expression patterns, which are similar (Fig. 6). The major trends are as follows: (1) $L h x 7$ is particularly conservative, (2) $L h \times 2 / 9$ are predominant in the telencephalon and conserved in prosomeres $\mathrm{p} 4$ and $\mathrm{p} 2$, (3) $x$-Lhxl/5 show restricted telencephalic expression but are conserved in $\mathrm{p} 3$ and $\mathrm{p} 1$ and label the zona limitans intrathalamica, and (4) there is exclusive expression of one of the subgroups in $\mathrm{p} 1-\mathrm{p} 4$, with alternating expression of the paralogs of a given subgroup. Strong similarities are therefore present between mouse and Xenopus and suggest homology between most of these areas.

Interestingly, the major differences between mouse and frog expression patterns happen to be located inside the telencephalon, the structure that is obviously the more divergent, particu- 

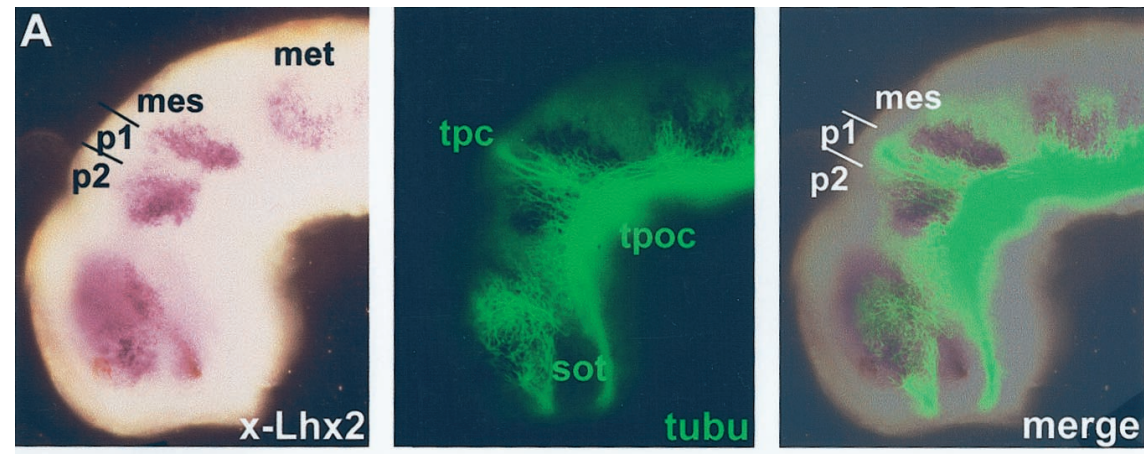

Figure 5. $x$-Lhx1 and $x$-Lhx2 expression relative to axonal tracts. $A, B$, Whole-mount lateral views of brains double labeled for $\alpha$-acetylated tubulin and $x$-Lhx2 $(A)$ or $x$-Lhx1 $(B)$ expression. The left panels show dark-field micrographs of in situ hybridization. The middle panels show tubulin immunofluorescence micrographs. The right panels show combined pictures. $t p c$, Tract of the posterior commissure; tpoc, tract of the postoptic commissure; sot, supraoptic tract; mes, mesencephalon; met, metencephalon. Note the striking correspondence and close relationships between early axon tracts and LIM-hd expression. The $t p c$ runs on the pretectal ( $p 1$ prosomere) band of $x$-Lhx1 expression, just between the two bands of $p 2$ and mesencephalic $x$-Lhx2 expression.
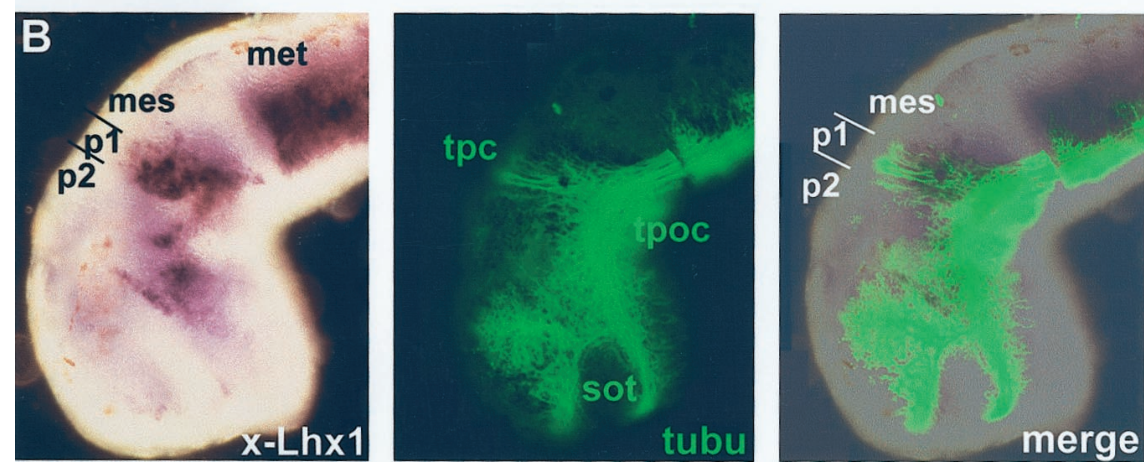

larly in terms of connectivity. An increasing number and complexity of forebrain connections were reached at the anamniote/ amniote transition, where two important innovations emerged (Striedter, 1997): a cortical relay of thalamic information and a massive palliosubpallial projection, resulting in a higher involvement of the cortex in the processing of sensory information. The fact that four studied LIM-hd members (Lhx1/2/5/9) are expressed in various patterns in the mouse pallium, whereas only two members (one paralog of each subgroup: $L h \times 2$ and $L h \times 5$ ) are present in the amphibian pallium, might be functionally representative of the higher complexity, increased connectivity, and higher involvement of the mammalian cortex in perception, elaboration of movements, and other integrated functions.

\section{LIM-hd genes define embryonic subdivisions in Xenopus telencephalon}

We suggest that LIM-hd expression allows to distinguish between telencephalic subdivisions in developing Xenopus. Such subdivisions defined by gene expression become more and more precise in the developing telencephalon of mice or birds (SmithFernandez et al., 1998; Puelles et al., 2000). In contrast, they are poorly known in frogs and fishes, probably because of the fact that their telencephalon is small and less differentiated (Hauptmann and Gerster, 2000). We suggest that stage 32 Xenopus telencephalon is delimited by a line drawn from the optic stalk and running dorsally orthogonal to the brain axis. Inside the telencephalon, concurrent expression of LIM-hd factors and other genes such as $x$-Dll3 defines pallial and subpallial compartments (Fig. 6D) (Papalopulu and Kintner, 1993). Inside these, LIM-hd expression defines two pallial and three subpallial divisions.

We suggest that the $x$-Lhx7-expressing area corresponds to the medial ganglionic eminence. The frog pallidum is histologically poorly delineated. Only connectivity and immunohistochemical data suggest the existence of a pallidum in amphibians (Marin et al., 1998b), but GABAergic neurons have never been found (for review, see Reiner et al., 1998). Our finding of an Lhx7-positive domain localized inside the distalless-positive subpallium is an additional excellent argument in favor of the existence of this structure. The mammalian mge also expresses Lhx6 and Lhx8 (two paralogs of $L h x 7 ; \operatorname{Lh} x 8$ is probably caused by a rodentspecific duplication) (Fig. 6A) (Failli et al., 2000). We do not know whether the frog mge expresses any other $x-L h x 7$ paralog. However, it is noteworthy that the mammalian mge also expresses Lhx2 and therefore presents a richer LIM-hd code. Functionally, members of the $L h \times 6 / 7 / 8$ group might be involved in the tangential migration of GABA interneurons from the mge to the striatum and cortex in rodents (Marin et al., 2000; Anderson et al., 2001). The $x$-Lhx7 expression pattern might suggest that similar migrations occur in the amphibian telencephalon. In another respect, $L h \times 2$ is strongly expressed in the proliferative zone of the rodent basal ganglia, which are hypoplasic in $L h x 2^{-1-}$ mice (Porter et al., 1997). The absence of $x$-Lhx2 in the Xenopus cell-poor pallidum therefore would agree with a role for $L h x 2$ in cell proliferation control.

Two other subdivisions, expressing $x$-Lhx2 and $x$-Lhx1/2/9, can be delineated from LIM-hd expression in Xenopus subpallium. Altogether, the three LIM-hd-deduced subpallial compartments might correspond to the three subdivisions proposed by Puelles et al. (2000) as the striatal, pallidal, and telencephalic stalk divisions of the basal forebrain. Among them, only the Lhx7-positive region can be attributed to the mge with some confidence. In the two other compartments the LIM-hd combinations are clearly different between Xenopus and mouse and might reflect the many differences in cell types and connectivity found in the basal ganglia of the two species (Reiner et al., 1998). However, we cannot exclude the possibility that cell migrations occur in frog telencephalon, as described in mouse, and could impair the interpretation of the results.

In the telencephalic pallium, four major subdivisions are found in birds and mammals: medial (hippocampus), dorsal (isocortex), lateral (olfactory cortex), and ventral (amygdala/claustrum) pallium (Puelles et al., 2000). These pallial divisions can be deduced from LIM-hd expression in mice, by comparing mediolateral 
xenopus

B

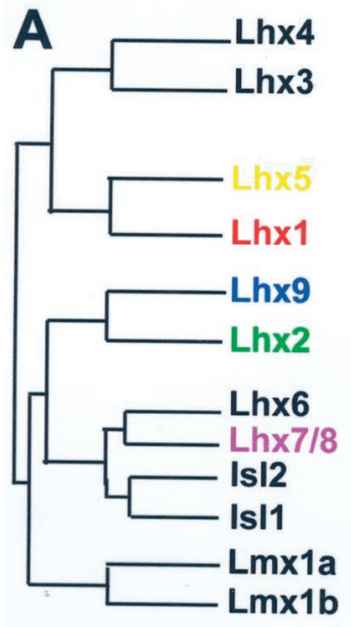

C

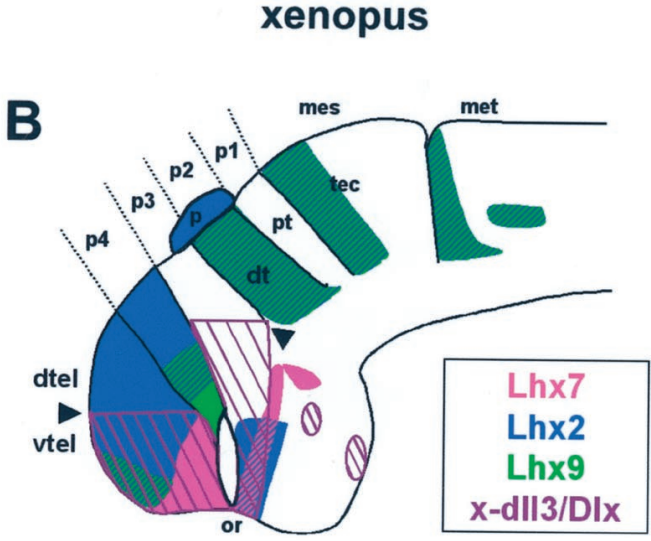

mouse
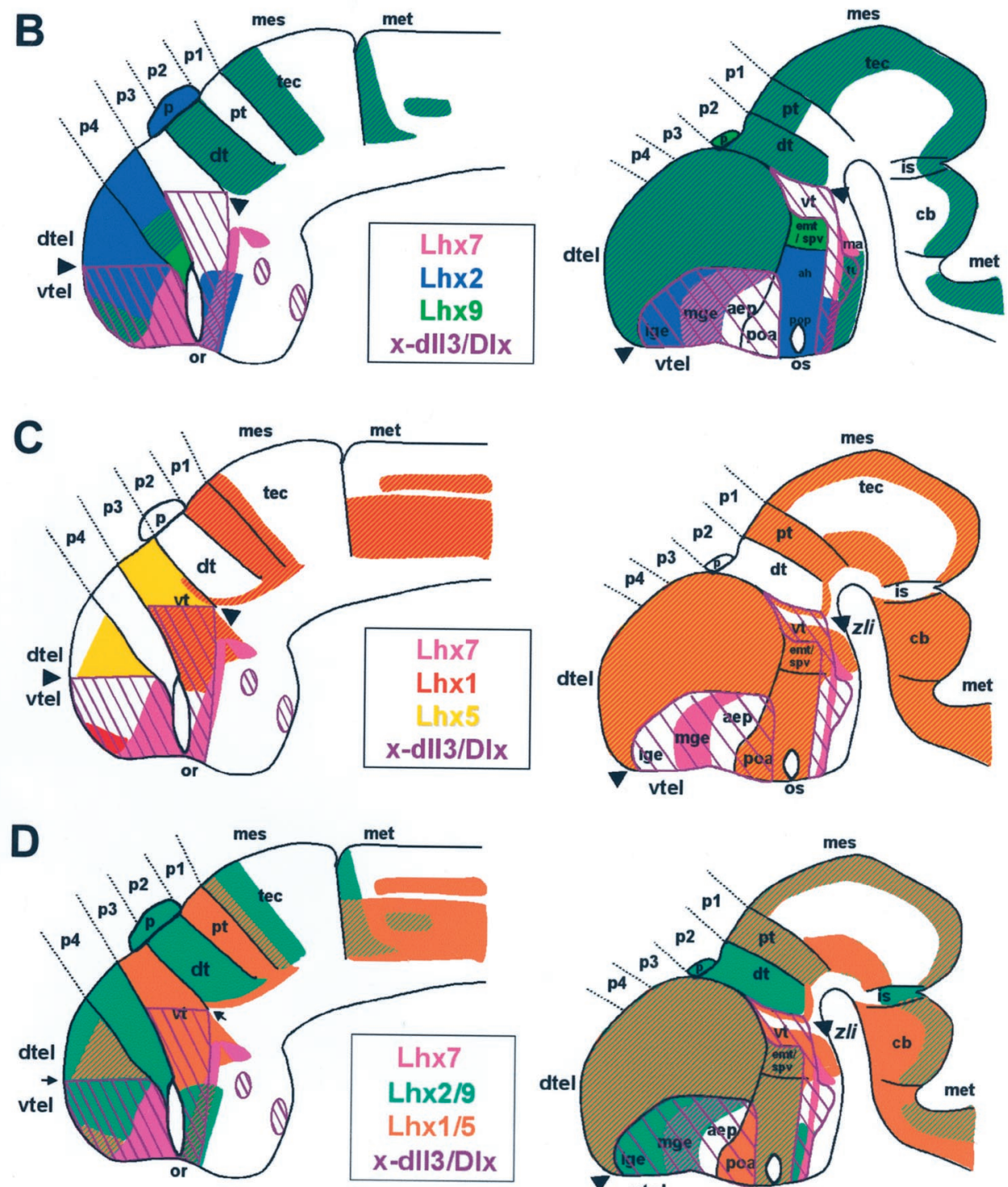
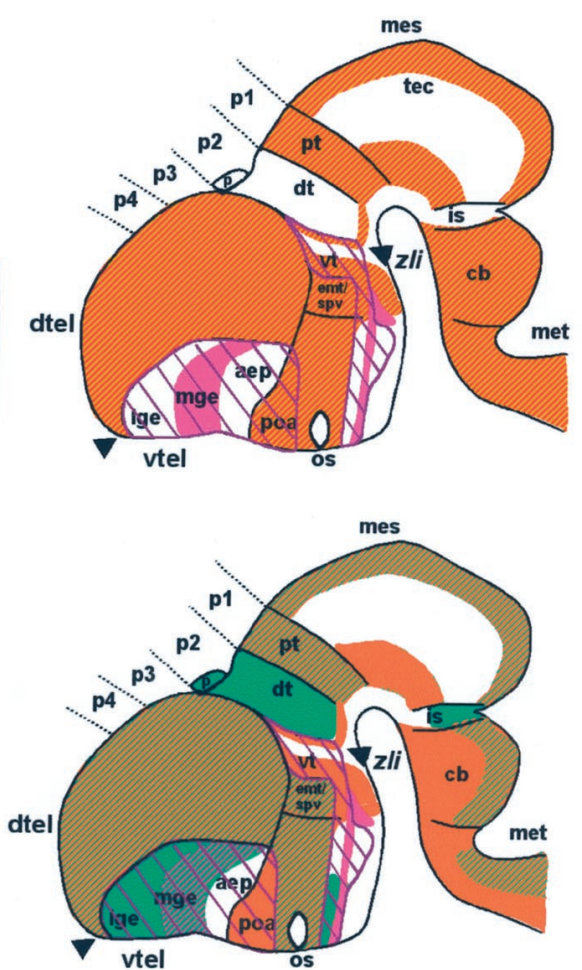

Figure 6. Schematic gene expression maps of Xenopus and mouse LIM-hd genes, with respect to subdivisions of the forebrain. A, A simplified phylogenetic tree of the LIM-hd family. The members studied in this paper are color coded, and their expression patterns in $B$ and $C$ are drawn in the same colors. $B-D$, Schematic color-coded (see boxes) recapitulations of Xenopus and mouse LIM-hd expression patterns, with reference to $x$-Dll3/mouse Dlx. In B, Lhx2 (blue)/9 (green) are compared with Lhx7 (pink) and $x$-Dll3/Dlx (purple). In C, Lhx1 (red)/5 (yellow) are compared with Lhx7 (pink) and $x$-Dll3/Dlx (purple). In D, the additive expressions of Lhx1/5 in orange and Lhx2/9 in turquoise are compared with Lhx7 (pink) and $x$-Dll3/Dlx ( purple). For abbreviations, see legend to Figure $1 D$.

extent and laminar patterns of Lhxl/2/5/9 expression (I. Bachy and S. Rétaux, unpublished observations). In Xenopus, only two pallial subdivisions were found: one expressing only $L h \times 2$, the other expressing $L h x 2 / 5$. Functionally, in mice $L h \times 2$ regulates the formation of the cortical hem (Bulchand et al., 2001), and Lhx5 controls neural patterning in the hippocampus (Zhao et al., 1999), implying crucial roles for the LIM-hd family in patterning pallial subdivisions. The presence in the small Xenopus pallium of two LIM-hd-defined compartments suggests that only two distinct functional areas are found in anamniotes, when using these specific markers. Smith-Fernandez et al. (1998) observed an intermediate territory between Dlx- and Emx-positive domains in frog telencephalon, which is likely to correspond to the ventral pallium defined by Puelles et al. (2000) in amniotes. This intermediate territory does not correspond to one of our LIM-hddefined compartments, because none of them is found in the Emx-negative, Dlx-negative portion of the frog telencephalon (our unpublished observations).

In conclusion, richer LIM-hd combinatorial expression in the mammalian pallium could reflect an enrichment in cortical connectivity. This will have to be functionally tested by overexpression experiments in Xenopus, or, conversely, by analyzing in these terms the mouse lines in which LIM-hd genes have already been inactivated.

Finally, the topological relationships of the deduced telencephalic subdivisions are organized along the anteroposterior axis 
of the brain, with the "basal ganglia" complex in the anterior position (apparently ventral, because of the brain curvature) and the pallium in a more caudal position (apparently dorsal). This appears strongly similar to the situation shown by SmithFernandez et al. (1998) for the chicken telencephalic fate map.

\section{LIM-hd expression defines conserved prosomeres in Xenopus diencephalon}

Prosomeres 1-4 show a remarkable and conserved alternation in expression of LIM-hd subgroups members between Xenopus and mouse. They can certainly be considered as prosomeric markers and should be taken into account for the definition of homologies in the diencephalon. Lhx1/5 label the pretectum (p1) and the ventral thalamus (p3), whereas $L h \times 2 / 9$ label the dorsal thalamus and epithalamus (p2) in both species. A paralog inversion apparently occurred between $\operatorname{Lh} x 2$ and $\operatorname{Lh} x 9$ in the pineal gland. This inversion might also be true for the rest of the diencephalon, although we have no way to verify this because the expressions of the two paralogs are identical in this region. Such inversions are relatively common (Derobert et al., 2000; Zerucha and Ekker, 2000) and are representative of the conservation of function and redundancy of developmental genes. Finally, Lhx1/5 conservatively label the $z l i$, a morphological landmark that divides dorsal and ventral thalamus and probably corresponds to a forebrain organizing center (Braun et al., 2000; Garda and Martinez, 2000). Overall, the conserved alternating, stripe-like, and exclusive expression of the $L h \times 1 / 5$ and $L h \times 2 / 9$ subgroups in the diencephalon suggests two remarks. First, this could imply negative interactions in the regulatory sequences of their promoters, which have been well conserved in tetrapods and would be interesting to analyze. Because the two subgroups are coexpressed in some regions of the telencephalon and posterior brain, it also suggests that different regulatory modules are used to promote their expression in different areas. Second, the diencephalic conservation of the LIM-hd code fits well with the fact that the pretectum, epithalamus, and thalamus are conservative features of vertebrate brains (Butler and Hodos, 1996). In particular, a lemnothalamus (receiving direct sensory inputs) and a collothalamus (receiving sensory inputs through tectal relay) can be distinguished in both amniotes and anamniotes. However, a collothalamic projection to the pallium is a new feature in amniotes, but the emergence of this major new pathway is not correlated with any variation in LIM-hd gene expression between frogs and mice.

\section{Concluding remarks}

Among other functions in brain development, LIM-hd family members work through interactions with LIM-specific cofactors to govern pathfinding events: LIM-hd combinatorial expression determines the topography of motorneurons axonal projections, and the genetic manipulation of the LIM-hd code results in predictable changes in their projections (Sharma et al., 1998; Thor et al., 1999; Kania et al., 2000). It is highly possible that a similar role is played in the forebrain, through recruitment of similar genetic cascades. In this respect, the detailed comparison of expression patterns of several LIM-hd members between species known to present well characterized differences in brain connectivity and neuronal types is useful in elaborating functional hypotheses. In summary, we show a degree of conservation of LIM-hd expression between Xenopus and mouse that strengthens the idea of conservation of brain patterning through vertebrate evolution. Interestingly, the divergences of expression observed in the telencephalon can be correlated with the emergence of new neuronal circuits that occurred at the anamniote/amniote transition. Additional anatomical studies on intermediate species (birds and reptiles) and functional analysis are needed to further analyze the role of the LIM-hd family in vertebrate brain development and evolution.

\section{REFERENCES}

Anderson SA, Marin O, Horn C, Jennings K, Rubenstein JLR (2001) Distinct cortical migration from the medial and lateral ganglionic eminences. Development 128:353-363.

Braun MM, Bermingham-McDonogh O, Roelink H (2000) The zona limitans intrathalamica as a signaling center in forebrain development. Soc Neurosci Abstr 26:22.3.

Bulchand S, Grove EA, Porter FD, Tole S (2001) LIM-homeodomain gene Lhx2 regulates the formation of the cortical hem. Mech Dev 100:165-175.

Butler AB, Hodos W (1996) Comparative vertebrate neuroanatomy. New York: Wiley.

Derobert Y, Germot A, Spengler T, Mazan S (2000) Structural and functional diversity of homeodomain genes of the orthodenticle and empty spiracles classes in Craniata. J Soc Biol 194:81-86.

Failli V, Rogard M, Mattei MG, Vernier P, Rétaux S (2000) Lhx9 and Lhx $9 \alpha$ LIM-homeodomain factors: genomic structure, expression patterns, chromosomal localization and phylogenetic analysis. Genomics 64:307-317.

Figdor MC, Stern CD (1993) Segmental organization of the embryonic diencephalon. Nature 363:630-634.

Fujii T, Pichel JG, Taira M, Toyama R, Dawid IB, Westphal H (1994) Expression patterns of the murine LIM class homeobox gene lim1 in the developing brain and excretory system. Dev. Dyn. 199:73-83.

Garda AL, Martinez S (2000) Zona limitans development and its role as a secondary organizer in prosencephalic regionalization. Soc Neurosci Abstr 26:22.2.

Grigoriou M, Tucker AS, Sharpe PT, Pachnis V (1998) Expression and regulation of $L h x 6$ and $L h x 7$, a novel subfamily of LIM homeodomain encoding genes, suggests a role in mammalian head development. Development 125:2063-2074.

Hauptmann G, Gerster T (2000) Regulatory gene expression patterns reveal transverse and longitudinal subdivisions of the embryonic zebrafish forebrain. Mech. Dev. 91:105-118.

Hobert O, Ruvkun G (1998) A common theme for LIM homeobox gene function across phylogeny? Biol. Bull. 195:377-380.

Jessel TM (2000) Neuronal specification in the spinal cord: inductive signals and transcriptional codes. Nat Rev 1:20-29.

Kania A, Johnson RL, Jessell T (2000) Coordinate roles for LIM homeodomain genes in directing the dorsoventral trajectory of motor axons in the vertebrate limb. Cell 102:161-173.

Lavdas AA, Grigoriou M, Pachnis V, Parnavelas G (1999) The medial ganglionic eminence gives rise to a population of early neurons in the developing cerebral cortex. J Neurosci 19:7881-7888.

Marin O, Smeets WJAJ, Gonzales A (1998a) Evolution of the basal ganglia in tetrapods: a new perspective based on recent studies in amphibians. Trends Neurosci 21:487-495.

Marin O, Smeets WJAJ, Gonzalez A (1998b) Basal ganglia organization in amphibians: chemoarchitecture. J Comp Neurol 392:285-312.

Marin O, Anderson SA, Rubenstein JLR (2000) Origin and molecular specification of striatal interneurons. J Neurosci 20:6063-6076.

Milan FJ, Puelles L (2000) Patterns of calretinin, calbindin, and tyrosine-hydroxylase expression are consistent with the prosomeric map of the frog diencephalon. J Comp Neurol 419:96-121.

Papalopulu N, Kintner C (1993) Xenopus Distalless related homeobox genes are expressed in the forebrain and are induced by planar signals. Development 117:961-975.

Pombal MA, Puelles L (1999) Prosomeric map of the lamprey forebrain based on calretinin immunocytochemistry, Nissl stain, and ancillary markers. J Comp Neurol 414:391-422.

Porter FD, Drago J, Xu Y, Cheema SS, Wassif C, Huang SP, Lee E, Grinberg A, Massalas JS, Bodine D, Alt F, Westphal H (1997) Lhx-2, a LIM homeobox gene, is required for eye, forebrain, and definitive erythrocyte development. Development 124:2935-2944.

Puelles L (1995) A segmental morphological paradigm for understanding vertebrate forebrain. Brain Behav Evol 46:319-337.

Puelles L, Rubenstein JLR (1993) Expression patterns of homeobox and other putative regulatory genes in the embryonic forebrain suggest a neuromeric organization. Trends Neurosci 11:472-479.

Puelles L, Kuwana E, Puelles E, Bulfone A, Shimamura K, Keleher J, Smiga S, Rubenstein JL (2000) Pallial and subpallial derivatives in the embryonic chick and mouse telencephalon, traced by the expression of the genes Dlx-2, Emx-1, Nkx-2.1, Pax-6, and Tbr-1. J Comp Neurol 424:409-438.

Reiner A, Medina L, Veenman CL (1998) Structural and functional evolution of the basal ganglia in vertebrates. Brain Res Rev 28:235-285. 
Rétaux S, Rogard M, Bach I, Failli V, Besson MJ (1999) Lhx9, a novel LIM homeodomain gene expressed in the developing forebrain. J Neurosci 19:783-793.

Sharma K, Sheng HZ, Lettieri K, Karavanov A, Potter S, Westphal H, Pfaff SL (1998) LIM homeodomain factors Lhx3 and Lhx4 assign subtype identities for motorneurons. Cell 95:817-828.

Sheng HZ, Bertuzzi S, Chiang C, Shawlot W, Taira M, Dawid IB, Westphal H (1997) Expression of murine Lhx-5 suggest a role in specifying the forebrain. Dev Dyn 208:266-277.

Smith-Fernandez A, Pieau C, Reperant J, Boncinelli E, Wassef M (1998) Expression of the Emx-1 and Dlx-1 homeobox genes defines three molecularly distinct domains in the telencephalon of mouse, chick, turtle and frog embryos: implications for the evolution of telencephalic subdivisions in amniotes. Development 125:2099-2111.

Striedter GF (1997) The telencephalon of tetrapods in evolution. Brain Behav Evol 49:179-213.

Taira M, Jamrich M, Good PJ, Dawid IB (1992) The LIM domain containing homeobox gene Xlim1 is expressed specifically in the organizer region of Xenopus gastrula embryos. Genes Dev 6:356-366.

Thor S, Andersson SGE, Tomlinson A, Thomas JB (1999) A LIMhomeodomain combinatorial code for motor neuron pathway selection. Nature 397:76-80.

Toyama R, Curtiss PE, Otani H, Kimura M, Dawid IB, Taira M (1995) The LIM class homeobox gene lim5: implied role in CNS patterning in Xenopus and zebrafish. Dev Biol 170:583-593.

Wullimann MF, Puelles L (1999) Postembryonic neural proliferation in the zebrafish forebrain and its relationship to prosomeric domains. Anat Embryol 199:329-348.

Zerucha T, Ekker M (2000) Distal-less-related homeobox genes of vertebrates: evolution, function, and regulation. Biochem Cell Biol 78:593-601.

Zhao Y, Sheng HZ, Amini R, Grinberg A, Lee E, Huang SP, Taira M, Westphal H (1999) Control of hippocampal morphogenesis and neuronal differentiation by the LIM homeobox gene Lhx5. Science 284 $1155-1158$. 